\title{
Clinical Impacts of Novel Coronavirus Disease 2019 on the Vital organs of the Human Body
}

\author{
Authors \\ Dr Farhan Ali ${ }^{1}$, Dr Adnan Bashir Bhatti, MD $^{2 *}$, Dr Rabia Kanwal ${ }^{3}$, Dr Hassan Orooj ${ }^{4}$ \\ ${ }^{1}$ Associate Physician (Internal medicine) Capital Hospital, Islamabad, Pakistan \\ ${ }^{2}$ Department of Internal Medicine, Capital Hospital, Islamabad, Pakistan \\ ${ }^{3}$ Post-Graduate Trainee, Combined Military Hospital, Rawalpindi, Pakistan \\ ${ }^{3}$ DG Health Services Islamabad, Pakistan \\ *Corresponding Author \\ Dr Adnan Bashir Bhatti, MD
}

Department of Internal Medicine, Capital Hospital, Islamabad, Pakistan

\begin{abstract}
Coronavirus Disease of 2019 (COVID19) was the first time diagnosed in the worker of the seafood market of Wuhan, a city of China, in December 2019 and now the entire world is facing its disastrous effects. The overall mortality rate per number of diagnosed COVID19 cases is 4.7 percent. It is highly contagious and infects all age groups but elderly people with co-morbid conditions are at high risk. There is variation in presentation from asymptomatic to critical condition. It attacks the vital organs of the body with features of severe pneumonia, acute respiratory distress syndrome (ARDS), cerebral edema, myocarditis, heart failure, renal failure, diarrhea, sepsis, septic shock, and death.

The assessment of the severity of the disease depends upon lymphopenia, thrombocytopenia, raised $C$ reactive protein $(C R P)$, raised serum ferritin levels, raised prolactin, deranged coagulation profile, and bilateral opacities infiltrates in the lungs. Confirmatory test for diagnosis is real-time PCR of nasal and pharyngeal swab in non-ventilatory patients. Hydroxychloroquine (previously used), remdesivir, lopinavir, ritonavir, interferon-beta, passive antibody therapy, and supportive care are available treatment options. We highlight in this review, background of the disease, important impacts on the major system of the body, transmission, diagnosis, treatment, and recommendations.
\end{abstract}

Keywords: Coronavirus, COVID19, Wuhan Disease, ARDS, Novel virus, Pandemic.

\section{Introduction and Background}

The world has been facing different viral infections since the origin of life. Now at this time highly lethal and contagious novel virus attacked the entire world and it became pandemic in a short period. The entire world is in a state of panic due to destroying effect of the novel Coronavirus Disease of 2019 (COVID19) on the infrastructure of health and economy. The first time COVID19 was identified in the workers of the seafood market of Wuhan province of China, in December 2019. The source of transmission of infection reported in humans through bat (96\%). The World health organization (WHO) declared the outbreak an international emergency and pandemic $^{[1]}$. 
The overall mortality in a per number of cases is 4.7 percent, ranging from 0.2 percent to 15 percent according to age group and other health problems till 28th March 2020 as compared with the mortality of flu pandemic in 1918 which was $3 \%$ to $5 \%{ }^{[2,3]}$.

The name Coronavirus is derived from the Latin word corona, which means "crown" or "halo", the virus has the characteristic appearance of the crown or solar corona under an electron microscope as the surface being covered in clubshaped protein spikes. It is an RNA virus belongs to Coronavirida and Orthocoronavirinae family and subfamily respectively.

There are six species of human coronaviruses, four of these strains are HCoV-229E, -NL63, OC43, and -HKU1. These strains circulate in the human population and cause mild respiratory infections in adults and children worldwide. The other three are beta coronavirus strains i.e. middle east respiratory syndrome-related coronavirus (MERS-CoV), severe acute respiratory syndrome coronavirus (SARS-CoV1), and severe acute respiratory syndrome coronavirus (SARS- CoV2) or COVID19 $9^{[4]}$.

The SARS-CoV1 was transmitted to humans from civet cats in 2002. The MERS-COV was transmitted to humans from the camel in 2012, and the SARS-CoV2 is a new strain of coronavirus that has not been previously identified in humans and is closely related genetically to the SARS-CoV1 hence the name given to this novel virus is SARS-CoV2 or COVID19 ${ }^{[5]}$.

\section{Strain of COVID19}

There are two types of strains of COVID19 identified in the genetic study of viruses. These are designated as $\mathrm{L}$ and $\mathrm{S}$ Strains. The $\mathrm{L}$ type $(70 \%)$ is more prevalent and has aggressive presentation and spread more quickly than $S$ type $(30 \%)$. The $\mathrm{L}$ type is more prevalent in the early stages of the outbreak in the Wuhan city of china, but the frequency decreased after early January 2020 on the other hand S type is less aggressive and evolutionarily older ${ }^{[6]}$.

\section{Vulnerable Age Group}

Elderly aged people are at high risk than middleaged and children. The few cases of involvement of children have been reported with mild to moderate symptoms. The suppressed immune system due to comorbid conditions is a high risk factor because the body does not have enough time to develop an immune response against the attacking pathogens, so mortality rate is very high in elderly patients with comorbid conditions, such as, chronic renal failure, hypertension, diabetes mellitus, coronary artery disease, connective tissue diseases, etc. Some aggressive presentation of COVID19 has been reported in younger age groups. The mechanism in these cases might be due to hyperactivity of the immune system that destroyed healthy cells alongside infected ones ${ }^{[7]}$.

\section{Clinical Presentations}

The presentation of the disease is highly variable; most patients are asymptomatic or present with mild to moderate and severe symptoms depending upon the underline comorbid conditions. Anosmia (loss of the sense of smell), low to high-grade fever, dry cough, sore throat, epigastric discomfort, diarrhea, easy fatigability, and myalgia are common symptoms in the most severe cases, severe pneumonia, acute respiratory distress syndrome (ARDS), sepsis, septic shock, myocarditis, renal failure, and multi-organ failure, all potentially leading to the mortality ${ }^{[1]}$.

COVID19 involves the vital organs of the body including the brain, lungs, heart, kidneys, intestines, and vessels due to the presence of ACE2 enzymes on the surface of these organs.

\section{Respiratory System}

The COVID-19 mainly affects the lower respiratory system through the accesses of enzyme ACE2 which is most abundant in type II alveolar cells of the lungs. The virus uses a special surface glycoprotein called a "spike" (peplomer) to adhere to ACE2 and enter into the cell ${ }^{[8]}$. The respiratory involvement presents with mild to moderate pneumonia, fever, fatigue, cough (with or without 
sputum production), anorexia, malaise, muscle pain, sore throat, nasal congestion, and/or headache.

Severe pneumonia presents with fever, cough, respiratory rate $>30$ breaths/min, severe respiratory distress, or $\mathrm{SpO} 2 \leq 93 \%$ in the room air.

\section{Acute Respiratory Distress Syndrome (ARDS)}

It is the type of respiratory failure characterized by widespread inflammation in the lungs within 1 week of a known clinical insult or new or worsening respiratory symptoms. Chest imaging shows bilateral opacities or infiltrates which are not explained by volume overload (on echocardiographic examination), lobar or lung collapse, or nodules. It is categorized based on oxygenation impairment without any risk factor:

- Mild ARDS: $200 \mathrm{mmHg}<\mathrm{PaO} 2 / \mathrm{FiO} 2$ a $\leq 300 \mathrm{mmHg}$ (with PEEP or CPAP $\geq 5$ cmH2O, or non-ventilated),

- Moderate ARDS: $100 \mathrm{mmHg}<\mathrm{PaO} 2 / \mathrm{FiO} 2$ $\leq 200 \mathrm{mmHg}$ (with PEEP $\geq 5 \mathrm{cmH} 2 \mathrm{O}$, or non-ventilated)

- Severe ARDS: $\mathrm{PaO} 2 / \mathrm{FiO} 2 \leq 100 \mathrm{mmHg}$ (with PEEP $\geq 5 \mathrm{cmH} 2 \mathrm{O}$, or non-ventilated); When $\mathrm{PaO} 2$ is not available, $\mathrm{SpO} 2 / \mathrm{FiO} 2 \leq$ 315 suggests ARDS.

\section{Cytokine Release Syndrome}

The cytokine storm is a life-threatening medical condition that occurred in the late stage of COVID19. The pathogenesis shows that SARSCoV2 virus enters the host cell through ACE2 receptors and control the DNA and lysosomes and start replication phase which ends with the rapture of the infective host cell, then the process of the inflammatory response is started. It is a form of systemic inflammatory syndrome which is started due to activation of a large number of white cells like helper $\mathrm{T}$ cells and macrophages that release inflammatory cytokines i.e. interleukin IL-6, interleukin IL-2, interleukin IL-12, TNF-alpha, interleukin-10, interferon-gamma, and granulocyte colony-stimulating factors. These cytokines are produced to regularize the inflammatory reactions ${ }^{[10]}$. In response to cytokine syndrome, characteristic changes appear in the body like altered mental level, weak pulse, cold extremities, low blood pressure, difficult or fast breathing, low oxygen saturation, decreased urine output along with coagulopathy, thrombocytopenia, acidosis, high lactate, or hyperbilirubinemia, and phenomena of sepsis start. Persisting hypotension despite volume resuscitation indicates septic $\operatorname{shock}^{[9]}$.

\section{Gastrointestinal System}

Gastrointestinal involvement presents with mild symptoms of epigastric discomfort, diarrhea, abdominal pain, nausea, and vomiting. The cause of involvement is due to abundantly presence of ACE2 enzymes on cell membranes of glandular cells of gastric, duodenal, and rectal epithelium as well as endothelial cells and enterocytes of the small intestine ${ }^{[11]}$. It has been reported that virus was found in the feces from one to twelve days or more, $53 \%$ cases had positives anal swab than oral swab in the later stages of infection while in $17 \%$ of cases virus was still in stool after the clearance from respiratory samples. There was a shift of source of reoccurrence from oral to anal in late stages $^{[11,12]}$.

\section{Renal System}

The COVID19 involves the renal system in late stages and presents with proteinuria, deranged renal functions, hematuria, and acute renal failure (ARF), computed tomography (CT) scan of the kidneys shows reduced renal density secondary to inflammation and edema ${ }^{[13,14]}$.

\section{Cardiac Involvement}

The cardiac involvement with COVID19 presents as myocarditis and heart failure due to the dissemination of the virus through the blood or the lymphatic system from the respiratory tract and triggering of inflammatory response. The increase of cardiac troponin levels, diffuse interstitial myocardial edema with hypokinesia and the slow 
gadolinium washout are indicators of cardiac injury ${ }^{[15]}$.

\section{Central Nervous System}

The involvement of the central nervous system (CNS) with COVID19 has been reported due to the expression of ACE2 receptors in endothelial linings of capillaries and neurons. The virus enters the brain via the transcribrial route (cribriform plate of the ethmoid bone) and through the systemic circulation. The altered sense of smell or hyposmia is an early feature and cerebral edema is a late feature. Severe CNS involvement alone can cause early death before systemic involvement. The findings of autopsies show that there were abundant of novel coronaviruses on the endothelium of cerebral microcirculation, cerebrospinal fluid, glial cells, and neuronal tissues $^{[16]}$.

\section{Hepatic Involvement}

There is mild derangement in liver function tests (LFTs) that are noted and the liver tissue displayed mild sinusoidal dilatation and minimal lymphocytic infiltration on liver biopsies. ALT, AST, GGT, Total bilirubin are significantly elevated in severe COVID19 but the liver is not the main target organ ${ }^{[17]}$.

\section{Hematological Involvement}

Lymphopenia is a characteristic feature and predictor of disease severity. It is reported that initially, lymphocyte decreased in severe cases then increased to higher than $10 \%$ until discharged, on the other hand, there was little decreased of lymphocytes at the onset of disease and higher than $20 \%$ at the time of recovery.

The pathophysiology of lymphopenia shows that the virus directly attacks the ACE2 receptor on the surface of lymphocyte and enters the cell and destroys it. It might be due to the disorder of inflammatory cytokines which cause lymphocyte apoptosis. Another mechanism of lymphopenia is hyperlactic acidosis which occurs during severe COVID19 and responsible for the suppression of lymphocyte proliferation ${ }^{[18]}$. Thrombocytopenia in the absence of primary etiology is also a bad prognostic sign it indicates the severity of disease and suggests serious organ malfunction and development of disseminated intravascular coagulation (DIC). The mechanism of thrombocytopenia in COVID19 is not confirmed.

It was suggested in the SARS-CoV-1 that thrombocytopenia was due to vast platelet consumption during aggregation at the site of damage of the endothelial lining of lungs by the virus. The second possibility is due to bone marrow suppression by infection, another reason may be pulmonary capillary bed damage due to infection which leads to a decrease in the release of platelets from mature megakaryocytes ${ }^{[19]}$.

\section{Transmission}

The transmission of COVID19 is through close contact, travel between areas, and by respiratory droplets during cough, sneeze, talk, and during fast breathing within a range of about 2 meters (6.6ft). People may also catch by touching a contaminated surface and then their face, eyes, and oral cavity. The virus may remain viable on plastic and steel for up to three days and cardboard for more than one day or on copper for more than four hours ${ }^{[2,4]}$. The coronaviruse is inactivated by soap, which destabilizes its bilayer. It is highly contagious when people are symptomatic, although spread may be possible during the incubation period which may range from 2 to 14 days $^{[2,20,21]}$.

\section{Laboratory Findings}

The most common laboratory findings in COVID19 are lymphopenia, neutrophilia, elevated AST, ALT, LDH, CRP, ferritin levels, and lactic acid. Elevated D-dimer and lymphopenia have been associated with high mortality. Procalcitonin is initially normal at the time of admission but may increase among critical cases ${ }^{[22]}$. 


\section{Chest Imaging}

The characteristic features in chest radiograph are bilateral non-homogenous opacities in late stages but initially, these are normal, High-resolution computed tomography (HRCT) chest typically demonstrate bilateral, peripheral ground glass opacities $^{[23]}$.

\section{Real-Time Polymerase Chain Reaction (PCR) Test}

The real-time polymerase chain reaction (PCR) test can identify SARS-CoV2 from a variety of clinical samples including nasopharyngeal swabs, throat swabs, bronchoalveolar lavages, bronchial washings, and sputum. The clinical data shows high sensitivity with bronchoalveolar lavage fluid $93 \%$, sputum $72 \%$, nasal swabs $63 \%$, fibro bronchoscope brush biopsy $46 \%$, pharyngeal swabs $32 \%$, feces $29 \%$, and blood $1 \%{ }^{[24]}$.

Bronchoalveolar lavage, the tracheal aspirate is only possible in ventilator patients. The swabs with wooden shafts should be used during nasopharyngeal or pharyngeal sampling and avoid calcium alginate swabs that inactivate some viruses and inhibit PCR testing. Place the swab sample in sterile tubes at $2-8 \mathrm{C}$ which is viable up to 72 hours after collection. If a delay in the testing sample it should be stored at $-70 \mathrm{C}$ or below. Only a nasopharyngeal sample can be used for the diagnosis of COVID19 ${ }^{[25]}$.

\section{Treatment}

There are few treatment options available against the novel COVID19. Chloroquine and Hydroxychloroquine (previously used) are used because of their anti-cytokine storm properties. Tocilizumab is an immunosuppressive drug and IL-6 antagonist which is mainly used in rheumatoid arthritis and severe form of juvenile arthritis also recommended for treatment. Another antiviral has been evaluated including remdesivir, lopinavir, ritonavir and interferon-beta in combination therapy ${ }^{[26]}$ There is no benefit of Chloroquine and Hydroxycholoroquine in preand post-exposure with COVID19 ${ }^{[27]}$.

\section{Passive Antibody Therapy}

The passive antibody therapy or non-vaccine immunization is another treatment option. In this technique plasma of recovered patient of COVID19 is used in critically ill patients, this plasma contains an abundance of antibodies specific to COVID19. These antibodies attach to the viral antigen present on the surface of targeting human cell than natural killer cells attach on antibodies and process of phagocytosis start, which is also known as antibody-dependent cellular cytotoxicity ${ }^{[28]}$.

\section{Discharge Criteria and Infectivity Period after Remission}

The patient should be discharged based on the afebrile state of more than three days, improved respiratory symptoms, and pulmonary imaging, Real-time PCR of the respiratory pathogen is negative twice consecutive (sampling interval $\geq$ 24 hours). The patient should be kept in isolation from community and family for 14 days after discharge and followed up on 2 and 4 weeks with $\mathrm{PCR}^{[29]}$.

The SARS-CoV2 virus can persist in upper respiratory samples for 7-12 days in moderate cases and up to 2 weeks in severe cases after remission. It persists in feces up to 4-5 weeks after onset. The test to confirm virus clearance in asymptomatic cases should be done after 14 days of an initial positive test. Serological tests of IgG antibodies are valuable in asymptomatic patients [30,31].

\section{Recommendations}

The COVID19 has produced a panic situation in the entire world - people think it is a token to death. No doubt the world is facing an extremely bad pandemic. Only precautionary measures can prevent the transmission of this lethal disease.

\section{Social Distancing and Hygienic Condition}

The transmission of disease can be decreased by frequent hand washing with soap including the back of hands, between fingers and under nails for 
20 seconds or use of $70 \%$ of alcohol-based sanitizer on regular basis in the absence of water. Cover mouth and nose with elbow or tissue paper when coughing and sneezing. Immediately discard used tissue paper. Social distancing is the only weapon in the fight for this lethal disease.

\section{Differentiation from other Respiratory Diseases and Anosmia}

The constitutional features of this disease have similarities with other respiratory diseases but fever, dry cough, and shortness of breath are distinguishing features. It should be differentiated from other upper and lower respiratory tract diseases based on the proper history of patients especially patients with a history of allergic asthma, COPD, bronchiectasis. Anosmia with respiratory involvement is an important clue of COVID19.

\section{Significance of Real-time PCR vs Antigen Antibodies Test}

Diagnosis should be made on real-time PCR tests as it is more reliable than the antigen-antibodies kit method. Antibodies IgM appear in the blood after 5-7 days of symptoms. Early diagnosis can be missed by the antigen-antibodies method and the community has to pay for it in the form of transmission of COVID19 in healthy people. It can be false-positive with some other infections due to cross-reactivity but it is very helpful by knowing the proper history of 5-7 days of symptoms and in asymptomatic patients.

\section{Antigen Test}

Although the antigen test is not available till now but workup should be done on it because it is the easiest and very cost-effective.

\section{Chest X-ray/HRCT Chest}

This test is a high value of significance in case of doubt of diagnosis based on PCR-negative and history of the patient. We can reduce mortality by early recognition and intervention.
6. Patients and Family Education upon Discharge

Education of patient and family members is very important in handling stool and urine due to prolonged periods of viral shedding in stool after remission.

\section{Precautions in the use of Self-Medications}

Education and counseling of community are required regarding the use of COVID19 medications without clinician advice to prevent severe complications associated with these drugs.

\section{Use of Immune-Boosting Diets}

The awareness among community should be produced for the use of diets containing vitamin $\mathrm{C}$, calcium, vitamin $\mathrm{D}$, zinc, etc; and the handling of animal products like milk and meat with proper hygienic protocols.

\section{References}

1. Coronavirus disease 2019.World Health Organization. Retrieved 15 March 2020.

2. Coronavirus (COVID-19) Mortality Rate. www.worldometers.info.5 March 2020.

3. Killingray, David; Phillips, Howard (2003). The Spanish Influenza Pandemic of 1918-1919: New Perspectives. Routledge.

4. Fan Y, Zhao K, Shi ZL, Zhou P.Bat Coronaviruses in China. Viruses. DOI:10.3390/v11030210.

5. Q\&A on coronaviruses. World Health Organization. 11 February 2020. Retrieved 24 February 2020.

6. Xiaolu Tang, Changcheng Wu, Xiang Li, Yuhe Song, Xinmin Yaoetal. On the origin and continuing evolution of SARS-CoV-2 National Science Review.DOI; org/10.1093/nsr/nwaa036.

7. Yuanyuan Dong, Xi Mo, Yabin Hu, Xin Qi, etal. Epidemiological Characteristics of 2143 Pediatric Patients With 2019 Coronavirus Disease in China, Pediatrics.DOI: 10.1542/peds.2020-0702. 
8. Letko $\mathrm{M}$, Marzi $\mathrm{A}$, Munster $\mathrm{V}$ (2020)."Functional assessment of cell entry and receptor usage for SARS-CoV-2 and other lineage B betacoronaviruses". Nature Microbiology. 5 (4): 562-569.

9. Clinical management of severe acute respiratory infection (SARI) when COVID-19 disease is suspected. Interim guidance 13 March 2020.world health organizations.

10. Yao $\mathrm{X}$, Ye $\mathrm{F}$, Zhang $\mathrm{M}$ etal. In Vitro Antiviral Activity and Projection of Optimized Dosing Design of Hydroxychloroquine for the Treatment of Severe Acute Respiratory Syndrome Coronavirus 2 (SARS-CoV-2). Clin. Infect.DOI: $10.1093 / \mathrm{cid} / \mathrm{ciaa} 237$.

11. Hamming, I.; Timens, W.; Bulthuis, M. L. C.; Lely, A. T.; Navis, G. J.; Goor, H. van Tissue distribution of ACE2 protein, the functional receptor for SARS coronavirus. A first step in understanding SARS pathogenesis. The Journal of Pathology. 2004. 203 (2):631-637.

12. GU, Jinyang; Han, Bing; Wang, Jian. COVID-19: Gastrointestinal manifestations and potential fecal-oral transmission. Journal Gastroenterology. DOI;10.1053/j.gastro.2020.02.054.

13. Cheng Y, Luo R, Wang K, et al. Kidney impairment is associated with in-hospital death of COVID-19 patients medRxiv DOI.10.1101/2020.02.18.20023242.

14. Li, Z., Wu, M., Guo, J. et al. Caution on kidney dysfunctions of 2019-nCoV patients. MedRxiv.DOI. 10.1101/2020.02.08.20021212.

15. Riccardo, M. Inciardi, MD1; Laura Lupi, MD1; Gregorio Zaccone, MD1; etal. cardiac Involvement in a Patient With Coronavirus Disease 2019 (COVID-19). JAMA Cardiol. DOI: 10.1001/jamacardio.2020.1096.

16. Abdul Mannan Baig, Areeba Khaleeq, Usman Ali, and Hira Syeda. Evidence of the COVID-19 Virus Targeting the CNS: Tissue Distribution, Host-Virus Interaction, and Proposed Neurotropic Mechanisms ACS Chemical Neuroscience 202011 (7), 995-998.

17. Wang $\mathrm{D}, \mathrm{Hu} \mathrm{B}$. $\mathrm{Hu}$ c, et al. Clinical characteristics of 138 hospitalized patients with 2019 novel corona infected pneumonia in Wuhan, China. JAMA. 2020; 323(11):1061-1069.

18. Li Tan, Qi Wang, Duanyang Zhangetal. Lymphopenia predicts disease severity of COVID-19: A descriptive and predictive study. Signal Transduction and Targeted Therapy.DOI 10.1038/s41392-020-0148-4.

19. Lippi G, Plebani M, Henry BM. Thrombocytopenia is associated with severe coronavirus disease 2019 (COVID19) infections: A meta-analysis Clinica Chimica Acta.2020, 13;506:145-148. doi: 10.1016/j.cca.2020.03.022.

20. Q \& A on COVID-19.European Centre for Disease Prevention and Control. Retrieved 23 March 2020.

21. Van Doremalen N, Bushmaker T, Morris DH, Holbrook MG, Gamble A, Williamson BN, et al .Correspondence: Aerosol and Surface Stability of SARSCoV-2 as Compared with SARS-CoV-1. NEJMed .DOI: 10.1056/NEJMoa2004973.

22. Guan WJ, Ni ZY, Hu Y, et al. Clinical Characteristics of Coronavirus Disease 2019 in china. NEJMed. DOI:10.1056/NEJMoa2002032.

23. Huang C, Wang Y, Li X, et al. Clinical features of patients infected with 2019 novel coronavirus in Wuhan, China. Lancet 2020; 395(10223):497-506

24. Wenling Wang, PhD1; Yanli Xu, MD2; Ruqin Gao, MD3; et Detection of SARSCoV-2 in Different Types of Clinical Specimens JAMA. DOI:10.1001/jama.2020.3786.

25. Interim Guidelines for Collecting, Handling, and Testing Clinical Specimens 
from Persons for Coronavirus Disease 2019 (COVID-19) Centers for Disease Control and Prevention March 25, 2020.

26. Kupferschmidt, Kai; Cohen, Jon (22 March 2020). WHO launches global megatrial of the four most promising coronavirus treatments. Science Magazine. DOI:10.1126/science.abb8497.

27. Severe Illness associated with using nonPharmaceutical Chloroquine Phosphate to Prevent and treat Coronavirus Disease 2019 (COVID-19)Centers for Disease Control and Prevention March 28, 2020.

28. Casadevall A, Pirofski LA.The convalescent sera option for containing COVID-19. JCI.130 (4): 15451548.

29. World Health Organization. Report of the WHO-China Joint Mission on Coronavirus Disease 2019 (COVID19). Geneva: WHO; 2020.

30. Young BE, Ong SWX, Kalimuddin S, Low JG, Tan SY, Loh J, et al. Epidemiologic features and clinical course of patients infected with SARS-CoV-2 in Singapore. JAMA. DOI: 10.1001/JAMA.2020.3204.

31. Cai J, Xu J, Lin D, Yang z, Xu L, Qu Z, et al. A case series of children with 2019 novel coronavirus infection: clinical and epidemiological features. Clin Infect Dis. DOI: $10.1093 /$ cid/ciaa198. 